\title{
Desafios e Recomendações à Atenção Oncológica durante a Pandemia da Covid-19
}

doi: https://doi.org/10.32635/2176-9745.RBC.2020v66nTemaAtual.1241

\author{
Challenges and Recommendations for Cancer Care during the COVID-19 Pandemic \\ Desafíos y Recomendaciones para la Atención del Cáncer durante la Pandemia de Covid-19
}

Chuade Cachoeira do Nascimento'; Pedro Henrique dos Santos Silva²; Sara Sabrina Vieira Cirilo3; Franciele Basso Fernandes Silva ${ }^{4}$

RESUMO

Introduçáo: A pandemia da doença causada pelo coronavírus 2019 (Covid-19) impactou o cotidiano da humanidade e do sistema de saúde mundial. Os pacientes oncológicos representam uma população de risco por apresentarem desfechos desfavoráveis quando infectados. Objetivo: O objetivo deste estudo foi avaliar as recomendaçôes para o manejo da população oncológica durante essa pandemia. Método: Utilizaram-se as plataformas PubMed e BVS para a seleção de artigos referentes ao manejo de pacientes com câncer no decorrer da pandemia da Covid-19, resgatando-se 16 publicaçôes que satisfizeram os critérios de inclusão e exclusão. Resultados: Todas as publicaçôes reforçaram a necessidade de estratégias específicas para o manejo dos pacientes oncológicos e a importância de medidas de prevenção contra a infecção pela síndrome respiratória aguda grave do coronavírus 2 (Sars-CoV-2). Os estudos discorreram sobre o tratamento ativo, cuidados paliativos e a saúde mental dos pacientes e profissionais de saúde. Apesar da sua importância, temas como o impacto futuro do atraso no diagnóstico e dos procedimentos cirúrgicos foram abordados em menos da metade dos estudos. Inexistem protocolos unanimes para o manejo dos pacientes oncológicos durante a pandemia. Conclusáo: $\mathrm{O}$ manejo ideal é equilibrar medidas preventivas ao contágio pelo vírus e estratégias para oferecer o melhor tratamento contra as neoplasias, considerando as características de cada caso, o bem-estar dos pacientes e dos profissionais de saúde, o impacto dos diagnósticos tardios e o acúmulo de cirurgias para o manejo atual e futuro dos pacientes oncológicos e do sistema de saúde.

Palavras-chave: Neoplasias/prevenção \& controle; Infecçôes por Coronavirus; Administração dos Cuidados ao Paciente; Pandemias.

\begin{abstract}
Introduction: The coronavirus disease 2019 (COVID-19) pandemic impacted the daily lives of humanity and the global health system. Cancer patients represent a population at risk for presenting unfavorable outcomes when infected. Objective: The objective of this study was to evaluate the recommendations for the management of the oncological population during this pandemic. Method: PubMed and VHL platforms were used to select articles related to the management of cancer patients during the COVID-19 pandemic, retrieving 16 publications that met the inclusion and exclusion criteria. Results: All publications reinforced the need for specific strategies for the management of cancer patients and the importance of preventive measures against the severe acute respiratory syndrome coronavirus 2 (SARS-CoV-2) infection. The studies discussed active treatment, palliative care and the mental health of patients and health professionals. Despite its importance, topics such as the future impact of delayed diagnosis and surgical procedures are addressed in less than half of the studies. There are no unanimous protocols for the management of cancer patients during the pandemic. Conclusion: The ideal management is to balance preventive measures against infection by the virus and strategies to offer the best treatment against neoplasms, considering the characteristics of each case, the well-being of patients and health professionals, the impact of late diagnoses and accumulation of surgeries for current and future management of cancer patients and the health system.
\end{abstract}

Key words: Neoplasms/prevention \& control; Coronavirus Infections; Patient Care Management; Pandemics.

\section{RESUMEN}

Introducción: La pandemia de la enfermedad del coronavirus 2019 (Covid-19) impactó la vida cotidiana de la humanidad y el sistema de salud global. Los pacientes con cáncer representan una población en riesgo de presentar resultados desfavorables cuando están infectados. Objetivo: El objetivo de este estudio fue evaluar las recomendaciones para el manejo de la población oncológica durante esta pandemia. Método: Se utilizaron las plataformas PubMed y VHL para seleccionar artículos relacionados con el manejo de pacientes con cáncer durante la pandemia de Covid-19, rescatando 16 publicaciones que cumplían con los criterios de inclusión y exclusión. Resultados: Todas las publicaciones reforzaron la necesidad de estrategias específicas para el tratamiento de pacientes con cáncer y la importancia de medidas preventivas contra la infección por el síndrome respiratorio agudo grave del coronavirus 2 (Sars-CoV-2). Los estudios analizaron el tratamiento activo, los cuidados paliativos y la salud mental de pacientes y profesionales de la salud. A pesar de su importancia, en menos de la mitad de los estudios se abordan temas como el impacto futuro del diagnóstico tardío y los procedimientos quirúrgicos. No existen protocolos unánimes para el manejo de pacientes con cáncer durante la pandemia. Conclusión: El manejo ideal es equilibrar las medidas preventivas contra la infección por el virus y las estrategias para ofrecer el mejor tratamiento contra la neoplasia, considerando las características de cada caso, el bienestar de pacientes y profesionales y el impacto de los diagnósticos tardíos y la acumulación de cirugías para el manejo actual y el futuro de los pacientes con cáncer y el sistema de salud.

Palabras clave: Neoplasias/prevención \& control; Infecciones por Coronavirus; Manejo de Atención al Paciente; Pandemias.

'Médica. Graduada em Medicina pela Faculdade de Medicina da Universidade Federal do Delta do Parnaíba (UFDPar). Parnaíba (PI), Brasil. Orcid iD: https:// orcid.org/0000-0002-3691-0474

${ }^{2}$ Acadêmico de Medicina da Faculdade de Medicina da UFDPar. Parnaíba (PI), Brasil. Orcid iD: https://orcid.org/0000-0001-6469-6076

${ }^{3}$ Fisioterapeuta. Graduação em Fisioterapia pela Universidade Federal do Piauí. Programa de Residência Multiprofissional em Saúde da Família da Universidade Estadual do Maranhão. Caxias (MA), Brasil. Orcid iD: https://orcid.org/0000-0002-7347-9927

${ }^{4}$ Doutora em Patologia pela Universidade Federal Fluminense (UFF). Professora-Adjunta do Curso de Medicina da UFDPar. Parnaíba (PI), Brasil. Orcid iD: https:// orcid.org/0000-0002-8617-0013

Endereço para correspondência: Pedro Henrique dos Santos Silva. Rua Duque de Caxias, 926 - Xique-Xique. Barras (PI), Brasil. E-mail: ph_beta@ufpi.edu.br 


\section{INTRODUÇÃO}

O ano de 2020 está sendo marcado pelo enfrentamento da pandemia da doença causada pelo coronavírus (do inglês, coronavirus disease 2019 - Covid-19). O relatório situacional divulgado no dia 21 de agosto de 2020 pela Organização Mundial da Saúde (OMS) demonstra um total de 22.492.312 casos da Covid-19 confirmados laboratorialmente em todo o mundo, espalhados por mais de 210 territórios, e 788.503 óbitos ${ }^{1}$. A Covid-19 é uma doença causada pela síndrome respiratória aguda grave do coronavírus 2 (do inglês, severe acute respiratory syndrome coronavirus 2 - Sars-CoV-2), descrita pela primeira vez na China ${ }^{2-4}$. O Sars-CoV-2 é um vírus de RNA de fita única de sentido positivo, envelopado, com 50-200 nm de diâmetro pertencente à família Coronaviridae 5 .

A Covid-19 é uma doença com altas taxas de transmissibilidade, que se dá, principalmente, por meio de aerossóis e superfícies contaminadas, embora existam relatos de transmissão por via oral-fecal ${ }^{6}$. Até o momento, não existe um tratamento específico para a Covid-19 e a melhor forma de combate essa doença é a adoção de medidas de prevenção à contaminação e à transmissão do vírus, como cuidados de higiene e distanciamento social ${ }^{7}$.

Os sintomas da Covid-19 incluem, principalmente, febre, tosse, distúrbios da respiração, e perda olfativa e de paladar temporariamente ${ }^{3}$. Apesar de se manifestar, na maioria das vezes, de forma assintomática, é esperado que $20 \%$ dos casos evoluam para formas mais graves da doença, especialmente em indivíduos que apresentem fatores de risco, como comorbidades (hipertensão arterial sistêmica, diabetes mellitus, neoplasias etc.), idade igual ou superior a 60 anos, transplantados e fumantes ${ }^{8,9}$.

Diante desse cenário, é fundamental lembrar que um dos principais problemas mundiais de saúde pública é o câncer, definido por um conjunto de mais de 100 doenças, que tem como característica comum o crescimento celular desordenado em decorrência de alteraçóes no código genético $^{10}$. Estimativas do Instituto Nacional de Câncer José Alencar Gomes da Silva (INCA) ${ }^{11}$ apontam que ocorrerão aproximadamente 625 mil casos novos de câncer no Brasil para cada ano do triênio 2020-2022.

Em virtude do estado de imunossupressão sistêmica causada por alguns tipos de câncer, bem como por suas formas de tratamento, como a quimioterapia, a radioterapia e o transplante de células, sabe-se que pacientes oncológicos são mais suscetíveis às infecçôes das vias respiratórias do que indivíduos sem neoplasias ${ }^{12,13}$. Estudos inicias sobre o impacto da infecção pelo Sars-CoV2 em pacientes com câncer ${ }^{14-16}$ apontam que essa população apresenta não apenas maior risco de infecção, mas também uma probabilidade maior de desfechos desfavoráveis, quando comparada à população geral, sendo uma população de risco e necessitando de atenção especial durante a corrente pandemia ${ }^{9,17,18}$.

Com base nas características do tratamento oncológico e nas alteraçōes originadas pela pandemia da Covid-19, faz-se necessária a construção de estratégias para minimizar o impacto desse período no bem-estar, diagnóstico, prognóstico e tratamento dos pacientes com câncer. Essas estratégias poderão servir de apoio não só no enfrentamento à Covid-19, mas para futuros ataques de doenças infecciosas. Diante do exposto, o presente estudo teve como objetivo avaliar as recomendaçóes para o manejo e o cuidado da população oncológica durante essa pandemia.

\section{MÉTODO}

Trata-se de uma revisão integrativa da literatura que abordou o tema "câncer e Covid-19", e utilizou artigos publicados nas bases de dados Medical Literature Analysis and Retrievel System Online (MEDLINE) por meio do motor de buscas PubMed e Biblioteca Virtual em Saúde (BVS).

A busca foi realizada no dia 5 de maio de 2020 e se concentrou em estudos publicados em língua inglesa, portuguesa ou espanhola, publicados nos últimos dois anos (2019 e 2020), e cuja versão integral estava disponível de forma gratuita. Os termos de busca utilizados na base da MEDLINE foram pesquisados no Medical Subject Headings (MeSH), e os utilizados na base da BVS foram pesquisados nos Descritores em Ciências da Saúde (DeCS).

As publicaçóes foram obtidas por meio de pesquisa em duas etapas para cada banco de dados, utilizando os descritores e operadores booleanos, conforme apresentado na Quadro 1. Para todos os descritores inseridos, também foram utilizadas suas variações nas línguas portuguesa e espanhola.

Após a recuperação dos artigos nessas etapas, foram realizadas a leitura e a avaliação de títulos e dos resumos, com posterior seleção por meio dos critérios de inclusão e exclusão, apresentados na Quadro 2. Os artigos selecionados em cada uma das plataformas foram avaliados e as duplicadas excluídas.

Na plataforma PubMed, foram recuperados 31 artigos na primeira etapa e 25 na segunda; dos quais, apenas 16 e 14 disponibilizavam suas versóes na íntegra e de forma gratuita, respectivamente. Após análise dos títulos e dos resumos, em conjunto com a aplicação dos critérios de inclusão e exclusão, foram captados oito e quatro estudos da primeira e da segunda etapas, respectivamente, resultando em 12 publicaçôes elegíveis nessa plataforma. 
Quadro 1. Etapas da busca no PubMed e BVS e descritores utilizados

\begin{tabular}{|c|c|c|c|c|c|}
\hline \multicolumn{6}{|c|}{ PubMed } \\
\hline Etapas & $\begin{array}{l}\text { Descritor } \\
\text { primário }\end{array}$ & $\begin{array}{l}\text { Operador } \\
\text { booleano }\end{array}$ & $\begin{array}{c}\text { Descritor } \\
\text { secundário }\end{array}$ & $\begin{array}{l}\text { Operador } \\
\text { booleano }\end{array}$ & $\begin{array}{l}\text { Descritor } \\
\text { terciário }\end{array}$ \\
\hline Primeira & Cancer & AND & COVID-19 & AND & Care \\
\hline Segunda & Cancer & AND & COVID-19 & AND & Management \\
\hline \multicolumn{6}{|c|}{ BVS } \\
\hline Etapas & $\begin{array}{l}\text { Descritor } \\
\text { primário }\end{array}$ & $\begin{array}{l}\text { Operador } \\
\text { booleano }\end{array}$ & $\begin{array}{c}\text { Descritor } \\
\text { secundário }\end{array}$ & $\begin{array}{l}\text { Operador } \\
\text { booleano }\end{array}$ & $\begin{array}{l}\text { Descritor } \\
\text { terciário }\end{array}$ \\
\hline Primeira & $\begin{array}{c}\text { Coronavirus } \\
\text { infections }\end{array}$ & AND & Neoplasms & AND & Care \\
\hline Segunda & $\begin{array}{c}\text { Coronavirus } \\
\text { infections }\end{array}$ & AND & Neoplasms & AND & Management \\
\hline
\end{tabular}

Quadro 2. Critérios de inclusão e de exclusão de artigos para compor este estudo

\begin{tabular}{|c|c|}
\hline Critérios de inclusão & Critérios de exclusão \\
\hline $\begin{array}{c}\text { 1.Artigos publicados } \\
\text { em qualquer época }\end{array}$ & $\begin{array}{c}\text { 1.Artigos em idiomas } \\
\text { diferentes: inglês, }\end{array}$ \\
pos idiomas inglês, & português e espanhol \\
2.Disponibilidade da & 2.Estudos em animais \\
versão completa & p.Estudos sem foco em \\
gratuita & ou no manejo/cuidado \\
3.Estudos com foco & com essa população, \\
na população de & com foco histológico/ \\
pacientes oncológicos & imunológico e/ou foco \\
e seu manejo durante & cirúrgico e/ou foco \\
a pandemia da & diagnóstico e/ou foco \\
Covid-19 (vírus Sars- & em outras infecções por \\
-Cov-2) & coronavírus diferentes \\
4.Artigos que tratem & do Sars-CoV-2 \\
de diretrizes/guias/ & 4.Artigos nos formatos \\
recomendações, & de relato de caso, \\
sendo editoriais, & correspondências, \\
comentários, artigos & revisões, comunicados \\
originais e relatórios & e cartas ao editor \\
\hline
\end{tabular}

Na base de dados BVS, foram recuperados 11 artigos na primeira etapa e 29 na segunda, com captação de cinco estudos em cada uma das etapas após avaliação dos títulos e dos resumos, e aplicação dos critérios de inclusão e exclusão. As dez publicações elegíveis encontravam-se em duplicidade, resultando em cinco publicações. Entre as publicaçóes recuperadas nas duas bases de dados (12 na PubMed e cinco na BVS), existia uma duplicidade que foi excluída. Dessa forma, 16 publicações foram lidas na íntegra e utilizadas nesta revisão.

Dos artigos selecionados, foram coletados dados referentes ao foco do artigo, país de elaboração, tipo de câncer abordado, tipos de tratamento e acompanhamento oncológico, tipos de medidas de prevenção à contaminação dos pacientes oncológicos pelo Sars-CoV-2, orientaçóes relacionadas ao tratamento oncológico, estratégias frente ao diagnóstico e à triagem de lesôes precursoras e cânceres em estágio inicial, estratégias frente ao manejo cirúrgico dos pacientes oncológicos, além das consideraçōes frente à saúde mental dos profissionais e de pacientes oncológicos.

Foi realizada análise exploratória sobre o banco de dados. As variáveis citadas anteriormente foram analisadas por estatística descritiva, estabelecendo-se a frequência e o percentual. A comparação entre as variáveis: consideraçôes frente à saúde mental dos profissionais da saúde e à saúde mental de pacientes oncológicos, bem como entre as abordagens diante do impacto do diagnóstico tardio e do impacto do adiamento de cirurgias, foi analisada pelos testes estatísticos qui-quadrado de Pearson e exato de Fisher em tabelas de contingência $2 \times 2$, com correção de continuidade. A análise descritiva serviu como base para a interpretaçáo dos resultados encontrados nos testes aplicados. Os testes foram realizados com auxílio do software IBM SPSS (versão 22, SPSS Inc., Chicago, Illinois, EUA). O nível de significância estatística adotado foi de $5 \%$.

\section{RESULTADOS}

No Quadro 3, estão detalhados os dados extraídos dos estudos selecionados, sendo eles: nomes dos autores, título, foco do estudo e local principal da sua realização.

De acordo com o local do estudo, 43,8\% eram do continente Asiático, 37,5\% do continente Europeu, $12,5 \%$ do Americano e 6,3\% do Africano. O país com maior número de publicaçôes foi a Itália, com um total de $31,3 \%$ ( $5 / 16$ estudos). Os estudos abordaram a população de pacientes oncológicos com câncer inicial (25\%); com doença avançada (50\%); em terapia antineoplásica ativa (100\%); em acompanhamento $(68,8 \%)$; em rastreio 
Quadro 3. Estudos utilizados na pesquisa e seus detalhes

\begin{tabular}{|c|c|c|c|}
\hline Autor/ano & Título & Foco & Local \\
\hline $\begin{array}{l}\text { Bitar et al. } \\
2020^{19}\end{array}$ & $\begin{array}{l}\text { The Lebanese Society of Medical } \\
\text { Oncology (LSMO) statement on the } \\
\text { care of patients with cancer during } \\
\text { the COVID- } 19 \text { pandemic }\end{array}$ & $\begin{array}{l}\text { Recomendações pragmáticas para o } \\
\text { atendimento diário de pacientes com } \\
\text { câncer durante a pandemia da Covid- } 19\end{array}$ & Líbano \\
\hline $\begin{array}{l}\text { Cortiula et al. } \\
2020^{20}\end{array}$ & $\begin{array}{l}\text { Managing COVID- } 19 \text { in the oncology } \\
\text { clinic and avoiding the distraction } \\
\text { effect }\end{array}$ & $\begin{array}{l}\text { Gerenciamento do tratamento } \\
\text { oncológico para evitar o efeito da } \\
\text { distração durante a pandemia da } \\
\text { Covid-19 }\end{array}$ & Itália \\
\hline $\begin{array}{l}\text { Curigliano et } \\
\text { al. } 2020^{21}\end{array}$ & $\begin{array}{l}\text { How to Guarantee the Best of Care } \\
\text { to Patients with Cancer During the } \\
\text { COVID-19 Epidemic: The Italian } \\
\text { Experience }\end{array}$ & $\begin{array}{l}\text { Orientações para manter o tratamento } \\
\text { de pacientes oncológicos durante a } \\
\text { pandemia da Covid-19 }\end{array}$ & Itália \\
\hline $\begin{array}{l}\text { Falandry et al. } \\
2020^{22}\end{array}$ & $\begin{array}{l}\text { Challenges with the management of } \\
\text { older patients with cancer during the } \\
\text { COVID- } 19 \text { pandemic }\end{array}$ & $\begin{array}{l}\text { Manejo de pacientes idosos oncológicos } \\
\text { durante a pandemia de Covid- } 19\end{array}$ & França \\
\hline $\begin{array}{l}\text { Jazieh et al. } \\
2020^{23}\end{array}$ & $\begin{array}{l}\text { Managing Oncology Services During } \\
\text { a Major Coronavirus Outbreak: } \\
\text { Lessons from the Saudi Arabia } \\
\text { Experience }\end{array}$ & $\begin{array}{l}\text { Abordagem usada para gerenciar } \\
\text { serviços oncológicos no surto mers-CoV } \\
\text { e a possibilidade de estrapolação para o } \\
\text { contexto atual - Covid- } 19\end{array}$ & $\begin{array}{l}\text { Arábia } \\
\text { Saudita }\end{array}$ \\
\hline $\begin{array}{l}\text { Kutikov et al. } \\
2020^{24}\end{array}$ & $\begin{array}{l}\text { A War on Two Fronts: Cancer Care in } \\
\text { the Time of COVID- } 19\end{array}$ & $\begin{array}{l}\text { Impacto da pandemia da Covid-19 no } \\
\text { tratamento oncológico e maneiras de } \\
\text { minimizá-lo }\end{array}$ & $\begin{array}{l}\text { Estados } \\
\text { Unidos }\end{array}$ \\
\hline $\begin{array}{l}\text { Lambertini et } \\
\text { al. } 2020^{25}\end{array}$ & $\begin{array}{l}\text { Cancer care during the spread of } \\
\text { coronavirus disease } 2019 \text { (COVID-19) } \\
\text { in ltaly: young oncologists' perspective }\end{array}$ & $\begin{array}{l}\text { Sugestões práticas para implementar } \\
\text { o tratamento do câncer durante a } \\
\text { pandemia da Covid- } 19\end{array}$ & Itália \\
\hline $\begin{array}{l}\text { Motlagh et al. } \\
2020^{26}\end{array}$ & $\begin{array}{l}\text { COVID } 19 \text { Prevention \& Care; A } \\
\text { Cancer Specific Guideline }\end{array}$ & $\begin{array}{l}\text { Recomendações e possíveis ações a } \\
\text { serem consideradas no diagnóstico } \\
\text { e tratamento do câncer durante a } \\
\text { pandemia da Covid-19 }\end{array}$ & Irã \\
\hline $\begin{array}{l}\text { Ngoi et al., } \\
2020^{27}\end{array}$ & $\begin{array}{l}\text { A segregated-team model to maintain } \\
\text { cancer care during the COVID- } 19 \\
\text { outbreak at an academic center in } \\
\text { Singapore }\end{array}$ & $\begin{array}{l}\text { Experiência com fluxo de trabalho } \\
\text { de equipe segregada para pacientes } \\
\text { oncológicos em resposta a Covid- } 19\end{array}$ & Cingapura \\
\hline $\begin{array}{l}\text { Porzio et al. } \\
2020^{28}\end{array}$ & $\begin{array}{l}\text { Home Care for Cancer Patients During } \\
\text { COVID-19 Pandemic: The Double } \\
\text { Triage Protocol }\end{array}$ & $\begin{array}{l}\text { Procedimentos e ferramentas utilizadas } \\
\text { em um serviço de atendimento } \\
\text { domiciliar a pacientes com câncer } \\
\text { durante a pandemia da Covid- } 19\end{array}$ & Itália \\
\hline $\begin{array}{l}\text { Romesser et } \\
\text { al. } 2020^{29}\end{array}$ & $\begin{array}{l}\text { Management of Locally Advanced } \\
\text { Rectal Cancer During The COVID-19 } \\
\text { Pandemic: A Necessary Paradigm } \\
\text { Change at Memorial Sloan Kettering } \\
\text { Cancer Center }\end{array}$ & $\begin{array}{l}\text { Novas diretrizes institucionais para o } \\
\text { tratamento de câncer retal durante a } \\
\text { pandemia da Covid- } 19\end{array}$ & $\begin{array}{l}\text { Estados } \\
\text { Unidos }\end{array}$ \\
\hline $\begin{array}{l}\text { Salako et al. } \\
2020^{30}\end{array}$ & $\begin{array}{l}\text { Upheaval in cancer care during the } \\
\text { COVID-19 outbreak }\end{array}$ & $\begin{array}{l}\text { Sugestões de intervenções para reduzir } \\
\text { o risco de transmissão da infecção } \\
\text { pela Covid-19 e manter o tratamento } \\
\text { entre pacientes com câncer durante a } \\
\text { pandemia }\end{array}$ & Nigéria \\
\hline $\begin{array}{l}\text { Shankar et al. } \\
\qquad 2020^{31}\end{array}$ & $\begin{array}{l}\text { Cancer Care Delivery Challenges } \\
\text { Amidst Coronavirus Disease - } 19 \\
\text { (COVID-19) Outbreak: Specific } \\
\text { Precautions for Cancer Patients and } \\
\text { Cancer Care Providers to Prevent } \\
\text { Spread }\end{array}$ & $\begin{array}{l}\text { Os desafios enfrentados pelos pacientes } \\
\text { oncológicos e seus cuidadores durante a } \\
\text { pandemia da Covid-19 e as precauções } \\
\text { necessárias }\end{array}$ & Índia \\
\hline
\end{tabular}


Quadro 3. continuação

\begin{tabular}{|c|c|c|c|}
\hline Autor/ano & Título & Foco & Local \\
\hline $\begin{array}{l}\text { Silvestris et al. } \\
2020^{32}\end{array}$ & $\begin{array}{l}\text { COVID-19 Pandemic and the Crisis } \\
\text { of Health Systems: The Experience of } \\
\text { the Apulia Cancer Network and of the } \\
\text { Comprehensive Cancer Center Istituto } \\
\text { Tumori "Giovanni Paolo II" of Bari }\end{array}$ & $\begin{array}{l}\text { Relato da experiência de uma rede } \\
\text { regional na capacidade de manutenção } \\
\text { do tratamento oncológico durante o } \\
\text { combate a Covid-19 }\end{array}$ & Itália \\
\hline Yusuf $2020^{33}$ & $\begin{array}{l}\text { Cancer care in the time of COVID- } \\
\text { 19-a perspective from Pakistan }\end{array}$ & $\begin{array}{l}\text { Impacto da infecção por coronavírus no } \\
\text { diagnóstico e tratamento do câncer }\end{array}$ & Paquistão \\
\hline $\begin{array}{l}\text { Zhao et al. } \\
2020^{34}\end{array}$ & $\begin{array}{l}\text { Recommendations of individualized } \\
\text { medical treatment and common } \\
\text { adverse events management for lung } \\
\text { cancer patients during the outbreak of } \\
\text { COVID-19 epidemic }\end{array}$ & $\begin{array}{l}\text { Recomendações e sugestões } \\
\text { de estratégias de tratamento e } \\
\text { gerenciamento de eventos adversos } \\
\text { comuns em pacientes com câncer } \\
\text { de pulmão durante a pandemia da } \\
\text { Covid-19 }\end{array}$ & China \\
\hline
\end{tabular}

inicial (43,8\%); em programação de intervenção cirúrgica $(68,8 \%)$, radioterapia $(50 \%)$ e quimioterapia $(75 \%)$; idosos (6,3\%). E tipos específicos de neoplasia, como câncer de pulmão $(25 \%)$, mama $(12,5 \%)$, próstata $(6,3 \%)$, pele $(6,3 \%)$, hematológicos $(12,5 \%)$, pâncreas $(6,3 \%)$ e retal $(6,3 \%)$.

Todos os estudos enfatizam que os pacientes oncológicos necessitam de estratégias específicas para o seu manejo durante a atual pandemia. Destes, 31,3\% apontam para o fato de os pacientes oncológicos apresentarem um maior risco de infecção pelo Sars-CoV-2. Em 81,3\%, fala-se em desfechos desfavoráveis para esses pacientes ao desenvolver a Covid-19, enquanto $87,5 \%$ destacam que a redistribuição de recursos de saúde pode dificultar a assistência a esses pacientes. Ainda, a maioria dos estudos $(81,3 \%)$ reforça que, durante a pandemia da Covid-19, o manejo dos pacientes com câncer deve encontrar o equilíbrio para manter o tratamento oncológico da melhor forma possível, minimizando o risco de infecção pelo Sars-CoV-2.

Todos os estudos reforçam a necessidade de adoção de medidas de prevenção à contaminação dos pacientes oncológicos pelo Sars-CoV-2, e as estratégias apontadas estão sintetizadas na Tabela 1.

Mais da metade dos estudos (62,5\%) levantam a existência de uma dificuldade significativa na construção de um protocolo universal em virtude das várias características de cada tipo de câncer, dos pacientes dos serviços de oncologia, o que faz com que, apesar da construção de recomendações e orientaçóes, exista a necessidade de uma avaliaçáo caso a caso para definir a melhor estratégia.

Dos estudos levantados, $87,5 \%$ apresentavam orientaçôes relacionadas aos tratamentos: sendo $81,3 \%$ para quimioterapia, $68,8 \%$ radioterapia, $37,5 \%$ imunoterapia e $18,8 \%$ hormonioterapia. A Tabela 2
Tabela 1. Medidas de controle da infecção por Covid-19 em pacientes oncológicos levantadas pelos estudos selecionados

\begin{tabular}{|c|c|c|}
\hline Medidas de prevenção & $\begin{array}{l}\mathbf{N}^{\circ} \text { de } \\
\text { estudos }\end{array}$ & $\%$ \\
\hline $\begin{array}{l}\text { Educação sobre higienização das } \\
\text { mãos }\end{array}$ & 7 & 43,8 \\
\hline Distanciamento social & 10 & 62,5 \\
\hline $\begin{array}{l}\text { Uso de EPI* pelos pacientes e } \\
\text { profissionais da saúde }\end{array}$ & 9 & 56,3 \\
\hline $\begin{array}{l}\text { Uso de EPI* pelos profissionais } \\
\text { da saúde }\end{array}$ & 7 & 43,8 \\
\hline $\begin{array}{l}\text { Realização de triagem de } \\
\text { pacientes para a Covid-19 }\end{array}$ & 12 & 75 \\
\hline $\begin{array}{l}\text { Realização de triagem de } \\
\text { profissionais para a Covid-19 }\end{array}$ & 6 & 37,5 \\
\hline $\begin{array}{l}\text { Limite de contato do paciente } \\
\text { com profissionais de saúde em } \\
\text { ambiente hospitalar e ambulatorial }\end{array}$ & 16 & 100 \\
\hline Utilização da telemedicina & 12 & 75 \\
\hline Tratamento por terapia oral & 10 & 62,5 \\
\hline Tratamento domiciliar & 5 & 31,3 \\
\hline $\begin{array}{l}\text { Limitação da realização de } \\
\text { procedimentos }\end{array}$ & 8 & 50 \\
\hline $\begin{array}{l}\text { Redução do número de visitantes } \\
\text { e/ou acompanhantes }\end{array}$ & 6 & 37,5 \\
\hline $\begin{array}{l}\text { Definição de local específico } \\
\text { para atendimento de pacientes } \\
\text { com Covid-19 ou suspeitos }\end{array}$ & 10 & 62,5 \\
\hline
\end{tabular}

Legenda: *Equipamentos de proteçấo individual.

resume as recomendaçôes dadas pelos estudos selecionados para o seguimento do tratamento oncológico, com objetivo de prevenção da infecção pela Covid-19.

Sete estudos $(43,8 \%)$ discorreram sobre o diagnóstico/ triagem de lesões precursoras e cânceres em estágio inicial. Os temas retratados incluíram: o impacto do atraso 
Tabela 2. Recomendações para o tratamento de pacientes oncológicos no período de pandemia pela Covid-19

\begin{tabular}{l|c|c}
\hline $\begin{array}{l}\text { Recomendações ao tratamento } \\
\text { oncológico }\end{array}$ & $\begin{array}{c}\mathbf{N}^{\circ} \text { de } \\
\text { estudos }\end{array}$ & $\%$ \\
\hline $\begin{array}{l}\text { Preferência por terapias mais } \\
\text { curtas }\end{array}$ & 7 & 43,8 \\
$\begin{array}{l}\text { Prioridade a terapias curativas } \\
\text { Alteração de terapias parenterais } \\
\text { feitas nos serviços de saúde para } \\
\text { terapias orais }\end{array}$ & 8 & 50 \\
$\begin{array}{l}\text { Alteração de terapias parenterais } \\
\text { feitas nos serviços de saúde para } \\
\text { terapias intravenosas domiciliares }\end{array}$ & 3 & 18,8 \\
$\begin{array}{l}\text { Adiamento ou atraso de terapias } \\
\text { que não comprometam o } \\
\text { controle da doença }\end{array}$ & 8 & 50 \\
$\begin{array}{l}\text { Suspensão do tratamento } \\
\text { em caso de dúvida dos reais } \\
\text { benefícios }\end{array}$ & 1 & 6,3 \\
$\begin{array}{l}\text { Preferência a terapias menos } \\
\text { imunossupressoras }\end{array}$ & 1 & 6,3 \\
$\begin{array}{l}\text { Suspensão de tratamentos } \\
\text { oncológicos ativos em pacientes } \\
\text { confirmados ou com suspeita de } \\
\text { Covid-19 }\end{array}$ & 1 & 6,3 \\
$\begin{array}{l}\text { Utilização de hormonioterapia } \\
\text { de manutenção em pacientes } \\
\text { apropriados }\end{array}$ & 1 & 6,3 \\
\hline
\end{tabular}

no diagnóstico e, consequentemente, no tratamento, acarretando a progressão da doença e a piora na sobrevida $(18,8 \%)$; o equilíbrio entre atrasar o diagnóstico e o risco da Covid-19 (25\%); a manutenção da consulta de triagem quando segura ou remarcá-la para o mais próximo possível $(18,8 \%)$; a suspensão da triagem de rotina $(18,8 \%)$; a construção de uma via/ rota terapêutica diagnóstica apropriada para reduzir o risco de infecção por Sars-CoV-2 (6,3\%); a informação ao paciente sobre os riscos e benefícios das intervençóes e a tomada de decisão compartilhada sobre o melhor manejo (6,3\%); e o estabelecimento de uma comissão supervisionada para avaliar caso a caso a hospitalizaçáo para realização de diagnóstico de caráter invasivo (6,3\%).

Em 68,8\% (11/16) dos estudos, o manejo cirúrgico dos pacientes oncológicos entrou em pauta, como: consideraçóes e preocupaçáo frente ao efeito imunossupressor da intervenção cirúrgica, causando uma maior suscetibilidade ao Sars-CoV-2 (31,3\%); impacto do atraso na realizaçáo do procedimento $(37,5 \%)$; adiamento da cirurgia eletiva em cânceres estáveis, menos agressivos ou paliativos (31,3\%); priorização da realização da cirurgia quando possível (12,5\%); reduçáo do período de recuperação pós-operatória em regime de internação hospitalar (6,3\%); e manutenção das cirurgias de emergência (6,3\%).

Quando analisada a preocupação com o impacto do adiamento de cirurgias e com a realizaçáo de diagnóstico de forma tardia, fatores que causam piora no prognóstico e/ou acúmulo futuro com sobrecarga dos serviços, notou-se que esse tema foi abordado em menos da metade dos estudos (37,6\%). Quando comparada a associação entre a abordagem desses dois temas, observou-se que apenas três $(18,8 \%)$ artigos abordaram ambos os temas, três $(18,8 \%)$ abordaram apenas o tema sobre o impacto do adiamento de cirurgias e dez (62,5\%) não abordaram os temas. Já outros três abordaram apenas o tema sobre o impacto do adiamento de cirurgias, demonstrando uma associação estatisticamente significativa $(\mathrm{p}=0,036)$.

Os cuidados paliativos foram abordados em $37,5 \%$ dos artigos: a necessidade de gestáo de pacientes em cuidado paliativo (31,3\%); o favorecimento de terapias curativas sobre as paliativas (6,3\%); a discussão com o paciente sobre riscos adicionais versus benefícios das linhas de terapia $(6,3 \%)$; o uso de telemedicina $(6,3 \%)$ e do atendimento domiciliar (6,3\%) no manejo; e o adiamento/ suspenção dos tratamentos com benefícios reduzidos na manutenção da qualidade de vida ou aumento da sobrevida, incluindo cirurgias $(18,8 \%)$.

Seis estudos $(37,5 \%)$ trazem consideraçôes em relação ao estado psicossocial dos pacientes oncológicos durante a pandemia da Covid-19, já que estes são obrigados a lidar não apenas com a carga do diagnóstico de câncer, mas também com as angústias e incertezas causadas pela pandemia (25\%), existindo, assim, a necessidade de estratégias para fornecer apoio psicológico a essa populaçáo, relatada em $18,8 \%$.

Em 31,3\% dos estudos, a telemedicina foi colocada como uma maneira de reduzir o impacto do isolamento social e manter uma via de comunicaçáo entre a equipe de saúde e o paciente para retirada de dúvidas e apoio. O impacto da atual da pandemia na saúde mental dos profissionais de saúde envolvidos no cuidado dos pacientes oncológicos foi pauta em seis estudos $(37,5 \%)$. Estes abordaram temas como: o aumento do medo, da angústia e da pressão entre os profissionais (18,8\%); a necessidade de apoio emocional aos profissionais durante esse período $(12,5 \%)$; o gerenciamento da equipe para minimizar o estresse e o esgotamento (18,8\%); a construção de estratégias para aumentar a moral dos profissionais $(12,5 \%)$, como compartilhamento de mensagens de agradecimento e informaçôes $(6,3 \%)$, além de sessôes diárias de aconselhamento (6,3\%); e o reforço do protocolo de prevençáo da Síndrome de Burnout (6,3\%). Seis estudos $(37,5 \%)$ não abordaram a saúde mental dos pacientes e dos profissionais de saúde. Cada tema foi 
tratado individualmente em quatro estudos $(25 \%) \mathrm{e}$, em apenas dois (12,5\%), ambos foram citados.

\section{DISCUSSÃO}

A pandemia da Covid-19 modificou o cotidiano da populaçáo mundial, afetando vários aspectos, como o econômico, o educacional, o social e o da saúde. Dessa forma, também houve alteração no manejo das pessoas enfermas. Pacientes portadores de neoplasias malignas hematológicas, pessoas em tratamentos que impactem na imunidade, como a quimioterapia, além de recém-operados e portadores de cânceres muito avançados, que apresentem grande debilidade física, fazem parte de uma populaçấo especial que apresenta condiçôes vulneráveis relacionadas à possibilidade de infecçôes virais, incluindo o Sars-CoV-2. Ademais, considerando os pacientes oncológicos em tratamento, a saturação do sistema de saúde, que está com os recursos voltados ao atendimento da pandemia, pode dificultar no tratamento pleno e íntegro da sua doença ${ }^{19-22,29,31,34}$. Dessa forma, durante esse período de pandemia, a mortalidade desses pacientes pode aumentar em razão da infecção e da não prestação de tratamento adequado ao câncer ${ }^{30,35,36}$.

A Covid-19 é uma nova doença e, portanto, carece de estudos que avaliem o seu impacto em populaçóes específicas. Liang et al. ${ }^{17}$ escreveram um dos primeiros estudos cujo objetivo era avaliar o impacto dessa doença na população de pacientes oncológicos, servindo de referência para diversos outros estudos, alguns dos quais estão presentes nessa revisão $0^{19-31,33}$. Os autores demonstraram que pacientes com câncer podem ser mais suscetíveis à infecção por Sars-CoV-2 e aos piores resultados da Covid-19, como a necessidade de internação em Unidade de Tratamento Intensivo (UTI) e de ventilação mecânica, servindo para demonstrar que esses pacientes necessitam de um acompanhamento mais minucioso, uma vez que seu quadro pode se deteriorar rapidamente.

O diagnóstico e o tratamento oncológico durante a atual pandemia devem ter como objetivo limitar os riscos causados pela infecção pelo vírus Sars-CoV-2 sem prejudicar a chance de cura de cada paciente, adaptando-se aos melhores recursos disponíveis ${ }^{23,24,29}$. Apesar dessa prerrogativa, sabe-se que, por conta da individualidade de cada tipo de câncer, das características de cada paciente e dos serviços de oncologia, é impossível estabelecer recomendações que possam ser aplicadas a todos de forma automatizada e, assim, cada caso deve ser avaliado individualmente em busca da melhor estratégia ${ }^{19,21,24-26,32,34}$.

A busca para manter o manejo adequando para o câncer e minimizar o risco de ter a Covid-19 esbarra nos fatos de que muitos dos tratamentos estão associados à imunodepressão e à escassez de estudos e de informaçóes sobre os desfechos de se optar por atrasar ou manter o tratamento habitual. Dessa forma, existem autores que recomendam a manutenção da programação cirúrgica ${ }^{21,26}$, enquanto outros defendem o seu adiamento ${ }^{20,25,31}$. Não há um consenso em relação à realização da quimioterapia e da radioterapia, apesar de a maioria dos estudos abordados nesta revisão relatar que, para alguns tipos de cânceres, pode se considerar o adiamento do início do tratamento.

O impacto negativo da pandemia nos pacientes oncológicos foi demonstrado em uma análise realizada com dados da Inglaterra, Irlanda do Norte e Estados Unidos, que estima que, no decorrer do próximo ano, se suceda um acréscimo no número de óbitos em paciente com câncer nesses países e que esse aumento ocorrerá não apenas em pacientes que contraírem a Covid-19, mas também naqueles que experimentarem atraso no diagnóstico e tratamento da neoplasia ${ }^{35}$. Além da realocaçáo dos recursos para o combate à pandemia, o receio de se expor ao vírus fará com que pacientes evitem procurar atendimento médico, o que pode comprometer a realizaçáo de diagnósticos e tratamentos oportunos ${ }^{36,37}$.

Apesar do impacto que diagnóstico tardio pode causar no futuro, como o avanço da neoplasia e o pior prognóstico, tal tema foi abordado em menos de um quinto das publicaçôes incluídas nesta revisão, demonstrando negligência frente a um tema de grandes repercussões. No entanto, a possibilidade de diminuir o risco para a infecção do Sars-CoV-2 em pacientes oncológicos por meio da recomendaçáo de medidas protetivas foi apontada em todos os estudos. Alguns estudos relatam que formas de reduzir o número de pacientes que frequentam serviços de saúde incluem a modificação de terapias intravenosas para orais e a aplicação de quimioterapia domiciliar quando possível, optar por ciclos mais curtos e espaçados de quimioterapia/radioterapias, adiar tratamentos que não modifiquem o controle da doença e realizar consultas e acompanhamentos por meio de telemedicina ${ }^{19,21-34}$. Dessa forma, estratégias já utilizadas no cuidado de alguns pacientes podem ser expandidas e priorizadas para um maior número de indivíduos, como é o caso da quimioterapia domiciliar. No entanto, como ressaltado por Falandry et al..$^{22}$, tal prática pode encontrar limitaçôes na necessidade de enfermagem domiciliar e no fornecimento de medicamentos.

A telemedicina, seja por meio de plataformas on-line de saúde ou de telefonemas, pode ser de grande apoio para o manejo dos pacientes em vários aspectos, como acompanhamento de pacientes sem a doença ativa, contato com o oncologista para retirada de dúvidas e aconselhamento, obedecendo às orientaçóes de distanciamento social e à monitorização dos efeitos 
colaterais do tratamento ${ }^{21-27,30,33,34}$, mas é necessário levar em conta que não se trata de uma soluçáo milagrosa, e que existem limitaçóes na esfera jurídica, no treinamento necessário para seu uso e na realização do exame físico ${ }^{38}$.

No cenário atual, em que não há uma diretriz consolidada e a decisão frente ao rumo do tratamento é complexa, se faz vital a participaçáo do paciente na tomada de decisão, devendo este ser informado sobre os riscos e benefícios dos esquemas disponíveis ${ }^{19,26}$. Alguns estudos não citam a participaçáo do paciente nesse processo, deixando-o a cargo do oncologista e/ou da equipe multidisciplinar, o que pode ter como consequência o agravamento de sintomas psíquicos e uma menor adesão ao tratamento ${ }^{23,25,29}$.

Quanto à saúde mental, de uma forma geral, os estudos já abordam que a atual pandemia, semelhante ao que aconteceu em surtos anteriores por outros vírus, aumentará os problemas relacionados com a saúde mental da populaçáo geral ${ }^{19-42} \mathrm{e}$, por conseguinte, dos pacientes oncológicos. Em razão da importância do tema e do impacto na saúde da população, a OMS divulgou um guia com cuidados para saúde mental durante a pandemia com dicas para a população em geral, agentes de saúde, líderes de equipe e supervisores em postos de saúde, cuidadores de crianças, idosos, cuidadores e pessoas com problemas de saúde e pessoas em isolamento ${ }^{43}$. Ao reportar o assunto à área da oncologia, o diagnóstico de câncer, por ser ainda estigmatizado e causar muitas dúvidas nos pacientes e familiares, tende a piorar as angústias dos pacientes em um cenário de tantas incertezas como o atual ${ }^{44}$. Apesar da importância desse tema, poucos dos estudos levantados abordaram a saúde mental dos pacientes oncológicos e o seu manejo durante esse período atípico ${ }^{22,24,25,30,33,34}$. Não se deve negligenciar esse aspecto durante o tratamento e é importante que se tenha em vista a necessidade de programas de suporte para pacientes, a fim de intervir adequadamente e da melhor maneira possível, podendo a telemedicina ser uma ferramenta de grande utilidade nessa área ${ }^{45}$.

Ainda sobre perturbaçóes na saúde mental, deve-se lembrar que os profissionais de saúde prestadores de cuidados aos pacientes oncológicos trabalham muitas vezes sobre pressão, o que pode causar estresse e repercutir na saúde mental e bem-estar psicossocial durante esse período e no futuro ${ }^{28,31,39,40,42,46} \mathrm{e}$, consequentemente, na qualidade do serviço prestado. Dessa forma, esses profissionais merecem uma atenção especial, com a adoção de medidas evitar o esgotamento ${ }^{27,33}$. Mesmo assim, somente alguns dos estudos captados nesta revisão abordaram esse tema ${ }^{23,25,27,28,31,33}$, o que pode demonstrar certa indiferença para com algo que pode ter um impacto significativo no manejo de pacientes oncológicos.
Outra faceta do manejo de pacientes oncológicos são os cuidados paliativos, com a premissa de gerar um bom controle dos sintomas e conforto ao paciente ${ }^{10}$. Assim, apesar do cenário atual, deve haver um esforço para sua realizaçáo, visando ao bem-estar do paciente, além de ser uma obrigação ética ${ }^{19,22,25,26,30}$. No entanto, os cuidados podem ser negligenciados em alguns cenários, no quais há a recomendação de se priorizar os com finalidade curativa $^{19}$.

Ensaios clínicos e outras pesquisas relacionadas ao câncer provavelmente sofrerão com a diminuição de iniciações e a desaceleração do ritmo de desenvolvimento, causando consequências não apenas momentâneas, mas também futuras ${ }^{21,24,25}$. Questôes éticas sobre as mudanças no manejo dos pacientes também devem ser levadas em conta $^{19}$. Um estudo muito importante foi o de Falandry et al. ${ }^{22}$, o qual aborda especificamente a população de pacientes oncológicos idosos durante a pandemia de Covid-19. Os autores enfatizam a necessidade da prevenção secundária nesses indivíduos para evitar complicaçōes como tromboembolismo venoso, eventos infecciosos relacionados a cateter, úlceras por pressão, quedas e delirium. Nesse estudo, recomenda-se ainda a realização da vacinação pneumocócica, quando disponível ${ }^{22}$.

As políticas e os procedimentos desenvolvidos e utilizados para lidar com a pandemia atual poderão servir para os próximos surtos infecciosos ou crises similares $^{24}$. Dessa forma, experiências prévias, como a relatada no estudo de Jazieh et al. ${ }^{23}$, sobre o manejo de pacientes oncológicos em um hospital durante um surto da síndrome respiratória do Oriente Médio causada pelo coronavírus mers (mers-CoV), são de grande valia. Os autores relataram a construção e a aplicação de um plano de gestáo de crise composto de cinco componentes principais: liderança e comunicação, gerenciamento de pacientes, gerenciamento de profissionais, controle de infecção e plano de recuperação, que se mostrou eficiente por reduzir o número de novos casos de mers-CoV a zero ${ }^{23}$.

As recomendaçóes listadas no presente estudo são baseadas no conhecimento atual e, dessa forma, podem evoluir à medida que novas pesquisas são realizadas. Não existe soluçáo única e fácil para gerir os cuidados oncológicos durante essa crise, além de náo se prever a sua duraçáo e real repercussão. Dessa forma, existe a necessidade de adaptação e evolução diante do cenário atual para cuidar dos pacientes da melhor forma possível.

\section{CONCLUSÃO}

Náo há protocolos unanimes para o manejo dos pacientes oncológicos durante a pandemia da Covid-19, 
mas o alvo das medidas propostas é equilibrar açóes de modo que evitem o contágio com o vírus, além de estabelecer estratégias para a oferta do melhor tratamento com objetivo terapêutico contra as neoplasias com os recursos disponíveis, sempre levando em conta a singularidade de cada caso e a saúde mental dos envolvidos, sejam pacientes, profissionais ou familiares.

Outra importante consideração é a necessidade de se pensar estratégias frente ao acúmulo de cirurgias e aos diagnósticos tardios para o câncer, a fim de evitar uma futura "pandemia" de cânceres mais agressivos e letais. Ademais, as recomendaçôes atuais deverão ser aprimoradas com o surgimento de novos estudos que servirão de apoio para o manejo do surto infeccioso corrente e de eventos futuros semelhantes.

\section{CONTRIBUIÇÕES}

Todos os autores contribuíram igualmente na concepção, análise e interpretação da pesquisa; na redação e revisão crítica com contribuição intelectual; e aprovaram a versão final a ser publicada.

\section{DECLARAÇÃO DE CONFLITO DE INTERESSES}

Nada a declarar.

\section{FONTES DE FINANCIAMENTO}

Não há.

\section{REFERÊNCIAS}

1. World Health Organization [Internet]. Geneva: WHO; c2020. WHO Coronavirus Disease (COVID-19) Dashboard; 2020 [cited 2020 Aug 22]. Available from: https://covid19.who.int

2. Rothan HA, Byrareddy SN. The epidemiology and pathogenesis of coronavirus disease (COVID-19) outbreak. J Autoimmun. 2020;109:102433. doi: https:// doi.org/10.1016/j.jaut.2020.102433

3. Sohrabi C, Alsafi Z, O'Neill N, et al. World Health Organization declares global emergency: a review of the 2019 novel coronavirus (COVID-19). Int J Surg. 2020;76:71-6. doi: https://doi.org/10.1016/j. ijsu.2020.02.034

4. Silva PHS, Cirilo SSV, Soares LS, et al. Déficit e ocupação de leitos de unidade de terapia intensiva adulto do Sistema Único de Saúde no estado do Piauí sob a ótica da COVID-19. Vigil Sanit Debate. 2020;8(3):61-9. doi: https://doi.org/10.22239/2317-269x.01606

5. Kakodkar P, Kaka N, Baig MN. A comprehensive literature review on the clinical presentation, and management of the pandemic coronavirus disease 2019 (COVID-19). Cureus. 2020;12(4):e7560. doi: https:// doi.org/10.7759/cureus.7560

6. Tang X, Wu C, Li X, et al. On the origin and continuing evolution of SARS-CoV-2. Natl Sci Rev. 2020;7(6):10223. doi: https://doi.org/10.1093/nsr/nwaa036

7. Kalil AC. Treating COVID-19-off-label drug use, compassionate use, and randomized clinical trials during pandemics. JAMA. 2020;323(19):1897-8. doi: https:// doi.org/10.1001/jama.2020.4742

8. Wu Z, McGoogan JM. Characteristics of and important lessons from the coronavirus disease 2019 (COVID-19) outbreak in China: summary of a report of 72314 cases from the Chinese Center for Disease Control and Prevention. JAMA. 2020;323(13):1239-42. doi: https:// doi.org/10.1001/jama.2020.2648

9. Zhang L, Zhu F, Xie L, et al. Clinical characteristics of COVID-19-infected cancer patients: a retrospective case study in three hospitals within Wuhan, China. Ann Oncol. 2020;31(7):894-901. doi: https://doi. org/10.1016/j.annonc.2020.03.296

10. Figueiredo EMA, Correia MM, Oliveira AF. Tratado de Oncologia. Vol. 1. Rio de Janeiro: Revinter; 2013. 981 p.

11. Instituto Nacional de Câncer José Alencar Gomes da Silva. Estimativa 2020: incidência de câncer no Brasil. Rio de Janeiro: INCA; 2019.

12. Yang G, Zhang H, Yang Y. Challenges and countermeasures of integrative cancer therapy in the epidemic of COVID-19. Integr Cancer Ther. 2020;19:1534735420912811. doi: https://doi. org/10.1177/1534735420912811

13. Kamboj M, Sepkowitz KA. Nosocomial infections in patients with cancer. Lancet Oncol. 2009;10(6):589-97. doi: https://doi.org/10.1016/S1470-2045(09)70069-5

14. Xia Y, Jin R, Zhao J, et al. Risk of COVID-19 for patients with cancer. Lancet Oncol. 2020;21(4):e180. doi: https://doi.org/10.1016/S1470-2045(20)30150-9

15. Wang H, Zhang L. Risk of COVID-19 for patients with cancer. Lancet Oncol. 2020;21(4):e181. doi: https://doi. org/10.1016/S1470-2045(20)30149-2

16. Onder G, Rezza G, Brusaferro S. Case-fatality rate and characteristics of patients dying in relation to COVID-19 in Italy. JAMA. 2020;323(18):1775-6. doi: https://doi. org/10.1001/jama.2020.4683

17. Liang W, Guan W, Chen R, et al. Cancer patients in SARS-CoV-2 infection: a nationwide analysis in China. Lancet Oncol. 2020;21(3):335-7. doi: https://doi. org/10.1016/S1470-2045(20)30096-6

18. Yu J, Ouyang W, Chua MLK, et al. SARS-CoV-2 transmission in patients with cancer at a tertiary care hospital in Wuhan, China. JAMA Oncol. 2020;6(7):1108-10. doi: https://doi.org/10.1001/ jamaoncol.2020.0980 
19. Bitar N, Kattan J, Kourie HR, et al. The Lebanese Society of Medical Oncology (LSMO) statement on the care of patients with cancer during the COVID-19 pandemic. Future Oncol. 2020;16(11):615-7. doi: https://doi. org/10.2217/fon-2020-0252

20. Cortiula F, Pettke A, Bartoletti M, et al. Managing COVID-19 in the oncology clinic and avoiding the distraction effect. Ann Oncol. 2020;31(5):553-5. doi: https://doi.org/10.1016/j.annonc.2020.03.286

21. Curigliano G. How to guarantee the best of care to patients with cancer during the COVID-19 epidemic: the italian experience. Oncologist. 2020;25(6):463-7. doi: https://doi.org/10.1634/ theoncologist.2020-0267

22. Falandry C, Filteau C, Ravot C, et al. Challenges with the management of older patients with cancer during the COVID-19 pandemic. J Geriatr Oncol. 2020;11(5):7479. doi: https://doi.org/10.1016/j.jgo.2020.03.020

23. Jazieh AR, Al Hadab A, Al Olayan A, et al. Managing oncology services during a major coronavirus outbreak: lessons from the Saudi Arabia experience. JCO Global Oncol. 2020;6:518-24. doi: https://doi.org/10.1200/ GO.20.00063

24. Kutikov A, Weinberg DS, Edelman MJ, et al. A war on two fronts: cancer care in the time of COVID-19. Ann Intern Med. 2020;172(11):756-8. doi: https://doi. org/10.7326/M20-1133

25. Lambertini M, Toss A, Passaro A, et al. Cancer care during the spread of coronavirus disease 2019 (COVID-19) in Italy: young oncologists' perspective. ESMO Open. 2020;5(2):e000759. doi: https://doi. org/10.1136/esmoopen-2020-000759

26. Motlagh A, Yamrali M, Azghandi S, et al. COVID19 prevention \& care; a cancer specific guideline. Arch Iran Med. 2020;23(4):255-64. doi: https://doi.org/10.34172/ aim. 2020.07

27. Ngoi N, Lim J, Ow S, et al. A segregated-team model to maintain cancer care during the COVID-19 outbreak at an academic center in Singapore. Ann Oncol. 2020;31(7):840-3. doi: https://doi.org/10.1016/j. annonc.2020.03.306

28. Porzio G, Cortellini A, Bruera E, et al. Home care for cancer patients during COVID-19 pandemic: the double triage protocol. J Pain Symptom Manage. 2020;60(1):e5-e7. doi: https://doi.org/10.1016/j. jpainsymman.2020.03.021

29. Romesser PB, Wu AJ, Cercek A, et al. Management of locally advanced rectal cancer during the COVID-19 pandemic: a necessary paradigm change at Memorial Sloan Kettering Cancer Center. Adv Radiat Oncol. 2020;5(4):687-9. doi: https://doi.org/10.1016/j. adro.2020.04.011

30. Salako O, Okunade K, Allsop M, et al. Upheaval in cancer care during the COVID-19 outbreak.
Ecancermedicalscience. 2020;14:ed97. doi: https://doi. org/10.3332/ecancer.2020.ed97

31. Shankar A, Saini D, Roy S, et al. Cancer care delivery challenges amidst coronavirus disease - 19 (COVID-19) outbreak: specific precautions for cancer patients and cancer care providers to prevent spread. Asian Pac J Cancer Prev. 2020;21(3):569-73. doi: https://doi. org/10.31557/APJCP.2020.21.3.569

32. Silvestris N, Moschetta A, Paradiso A, et al. COVID-19 Pandemic and the crisis of health systems: the experience of the Apulia Cancer Network and of the Comprehensive Cancer Center Istituto Tumori "Giovanni Paolo II" of Bari. Int J Environ Res Public Health. 2020;17(8):2763. doi: https://doi.org/10.3390/ijerph17082763

33. Yusuf A. Cancer care in the time of COVID-19-a perspective from Pakistan. Ecancermedicalscience. 2020;14:1026. doi: https://doi.org/10.3332/ ecancer.2020.1026

34. Zhao Z, Bai H, Duan J, et al. Recommendations of individualized medical treatment and common adverse events management for lung cancer patients during the outbreak of COVID-19 epidemic. Thorac Cancer. 2020;11(6):1752-7. doi: https://doi.org/10.1111/17597714.13424

35. Wise J. Covid-19: cancer mortality could rise at least $20 \%$ because of pandemic, study finds. BMJ. 2020;369:m1735. doi: https://doi.org/10.1136/bmj.m1735

36. Lai AG, Pasea L, Banerjee A, et al. Estimating excess mortality in people with cancer and multimorbidity in the COVID-19 emergency. MedRxiv [Preprint]. 2020 June 1. doi: https://doi.org/10.1101/2020.05.27.20083287

37. Cirilo SSV, Silva PHS, Cruz VT, et al. Necessidade de assistência psicossocial em tempos de pandemia causada pelo novo coronavírus: um olhar atento aos pacientes oncológicos e aos profissionais da área da oncologia. Rev Bras Cancerol. 2020;66(TemaAtual):e-1071. doi: https://doi.org/10.32635/2176-9745. RBC.2020v66nTemaAtual.1071

38. Al-Shamsi HO, Alhazzani W, Alhuraiji A, et al. A practical approach to the management of cancer patients during the novel coronavirus disease 2019 (COVID-19) pandemic: an international collaborative group. Oncologist. 2020;25(6):e936-e945. doi: https:// doi.org/10.1634/theoncologist.2020-0213

39. Torales J, O'Higgins M, Castaldelli-Maia JM, et al. The outbreak of COVID-19 coronavirus and its impact on global mental health. Int J Soc Psychiatry. 2020;66(4):31720. doi: https://doi.org/10.1177/0020764020915212

40. Mukhtar S. Psychological health during the coronavirus disease 2019 pandemic outbreak. Int J Soc Psychiatry. 2020;66(4):512-6. doi: https://doi. org/10.1177/0020764020925835

41. Fekih-Romdhane F, Ghrissi F, Abbassi B, et al. Prevalence and predictors of PTSD during the COVID-19 
pandemic: findings from a Tunisian community sample. Psychiatry Research. 2020;290:113131. doi: https://doi. org/10.1016/j.psychres.2020.113131

42. Rajkumar RP. COVID-19 and mental health: a review of the existing literature. Asian J Psychiatr. 2020;52:102066. doi: https://doi.org/10.1016/j.ajp.2020.102066

43. World Health Organization. Covid-19: OMS divulga guia com cuidados para saúde mental durante pandemia [Internet]. ONU News. 2020 mar 18 [acesso 2020 ago 22]. Disponível em: https://news.un.org/pt/ story/2020/03/1707792

44. Cure Today [Internet]. Ellesmere Port, UK: Cure; [2002]. [Video], How COVID-19 has impacted anxiety, mental health in patients with cancer; 2020 May 12 [cited 2020 Aug 22]; [5 min., 6 sec]. Available from: https://www. curetoday.com/view/how-covid19-has-impacted-anxietymental-health-in-patients-with-cancer

45. Instituto Oncoguia [Internet]. Sáo Paulo: Instituto Oncoguia; c2003-2020. Lidando com a ansiedade durante a pandemia do coronavírus; 2020 abr 6 [acesso 2020 ago 22]. Disponível em: http://www.oncoguia.org. $\mathrm{br} /$ conteudo/saude-emocional/13513/1204/

46. Greenberg N, Docherty M, Gnanapragasam S, et al. Managing mental health challenges faced by healthcare workers during covid-19 pandemic. BMJ. 2020;368:m1211. doi: https://doi.org/10.1136/bmj. $\mathrm{m} 1211$ 